[0212-7199 (2005) 22:2: pp 55-58] ANALES DE MEDICINA INTERNA Copyright (C) 2005 ARAN EDICIONES, S.L.

AN. MED. INTERNA (Madrid) Vol. 22, N. ${ }^{\circ} 2$, pp. 55-58, 2005

\title{
Estudio de la influencia del consumo de lácteos fermentados en una población de pacientes alérgicos
}

\author{
D. A. DE LUIS, A R. SANTAMARÍA, M. GONZÁLEZ SAGRADO, O. IZAOLA, \\ A. ARMENTÍA, R. ALLER
}

Unidad de Apoyo a la Investigación. Sección de Endocrinología y Nutrición. Hospital Universitario Río Hortega. Instituto de Endocrinología y Nutrición. Facultad de Medicina. Valladolid

\author{
STUDY OF INFLUENCE OF DIETARY YOGURT IN AN ALLERGIC \\ POPULATION
}

\section{RESUMEN}

Fundamento: La prevalencia de enfermedades alérgicas ha aumentado en las últimas décadas en los países desarrollados, por lo que se están ensayando nuevas alternativas terapéuticas. Los efectos beneficiosos de los probióticos han sido descritos en algunas patologías, especialmente en las diarreas, ciertos tumores y la sintomatología de la alergia, por lo que están adquiriendo una gran importancia por las aplicaciones en el manejo preventivo y terapéutico de esta patología. El objetivo de nuestro estudio fue describir la situación nutricional y la influencia de la ingesta de productos lácteos fermentados en una población de pacientes alérgicos.

Pacientes y métodos: Se seleccionaron 44 pacientes alérgicos, se les tomó los siguientes datos: edad, sexo, lugar de residencia; se les realizó una valoración antropométlica y una encuesta nutricional de 7 días, asî como la determinación de variables aiérgicas (rash cutáneo, niveles séricos de IgE y número de crisis al año).

Resultados: Dieciocho pacientes $(40,9 \%)$ eran mujeres y $26(59,1 \%)$ varones con una media de edad de $24,5(10,3)$ años. La mayoría de los pacientes $(20,5 \%)$ se encuentra en el P25-50 tanto para el pliegue tricipital, como circunferencia del brazo y circunferencia muscular del brazo lo que indica que mantienen dentro de la normalidad los compartimentos graso y proteico muscular. Con respecto al consumo de lácteos fermentados, 16 pacientes consumían estos productos habitualmente $(27,1 \%)$, con una media de tomas a la semana de $1,79(3,16)$ y una cantidad total a la semana de (mg-ml) $(593,4(461,9)$. Con respecto a las variables alérgicas, el número de crisis/año fue de $1,44(1,8)$, los valores medios de IgE séricos fueron de 35,68 (3 1,93) UI/L y con un valor medio del rash cutáneo de 10,09 $(2,8) \mathrm{mm}$. Los pacientes que consumían más de $593 \mathrm{mg} / \mathrm{semana}$ de lácteos fermentados presentaban menor diámetro en el rash cutáneo $[10,12(0,8) \mathrm{mm}$ vs $8,85(1,1) \mathrm{mm} ; \mathrm{p}<0,05]$, sin encontrar diferencias en el resto de variables alérgicas.

Conclusión: El consumo de lácteos fermentados en pacientes alérgicos puede ser beneficioso, disminuyendo determinados marcadores alérgicos. No obstante, son necesarios estudios con mayor número de pacientes y de intervención para evaluar esta influencia sobre variables clínicas de mayor relevancia.

PALABRAS CLAVE: Alergia y probióticos.

\section{ABSTRACT}

Background: Prevalence of allergic diseases has been increased in last years; new alternative therapies have been employed. Beneficial effects of probiotics have been described in some pathologies such as tumors, diarrhea, and allergic disease. The aim of our work was to describe nutritional status and influence of probiotic consumption in allergic population

Patients and methods: 44 allergic patients were selected, in all patients were recorded; sex, age, place of residence, anthropometric evaluation, dietary questonnaire and allergic variables (rush, blood levels of Ig E, and number of crisis in a year).

Results: 18 patients (40.9\%) were females and 26 (59.1\%) males with an average age of 24.5 (10.3) years. Most of patients (20.5\%) are located in percentil P25-50, showing a good nutritional status. 16 patients consumed probiotics $(27,1 \%)$, with an average of consumption per week 1.79 (3.16) and an average amount per wee ( $\mathrm{mg}$ - $\mathrm{ml})(593.4$ (461.9). The number of crisis year was 1.44 (1.8), average value of Ig $E$ was 35.68 (31.93) UI/L and rush 10.09 (2.8) mm. Patiens with a consumption of probiotics higher than $593 \mathrm{mg} /$ week showed a IOW rush diameter $(10,12(0,8) \mathrm{mm}$ vs $8.85(1.1) \mathrm{mm} ; \mathrm{p}<0.05)$, without statistical differences in other valiables.

Conclusion: Consumption of probiotics in allergic patients could be benefitial. Further studies with more patients and intervention designs will be necesary to analyze this relations.

KEY WORDS: Allergy and probiotics.

De Luis DA, Santamaría AR, González Sagrado M, Izaola O, Armentía A, Aller R. Estudio de la influencia del consumo de lácteos fermentados en una población de pacientes alérgicos. An Med Interna (Madrid) 2005; 22: 55-58.

Trabajo aceptado: 19 de octubre de 2004

Correspondencia: D. A. de Luis Román. C/ Los Perales, 16. Las Aceñas. 47130 Simancas. Valladolid. 


\section{INTRODUCCIÓN}

Las enfermedades alérgicas son reacciones imnunológicas mediadas por Ig E frente a alérgenos a nivel de mucosas (nasofaríngea, conjuntival, bronquial) y de la piel. Siendo las manifestaciones clínicas más frecuentes: rinitis, asma, edema de glotis, eczema atópico, ulticaria o anafila;ia. Un alérgeno es un antígeno capaz de inducir una respuesta mediada por Ig E. Generalmente son proteínas o glucoproteínas; los principales son: ácaros, epitelios, pólenes, mohos (1).

Los probióticos son microorganismos vivos (principalmente bacterias y levaduras) que son agregados como suplemento en las dietas y que afectan en forma beneficiosa al desarrollo de la flora microbiana en intestino (definición de R. Fuller en 1990) (2). Para considerar un microorganismo como probiótico debe ser de origen humano, aislado del tracto gastrointestinal, no ser patógeno para el hombre, sobrevivir a la acidez gástrica y al paso por la zona proximal del tracto gastrointestinal, ser estable y no conjugable con ácidos y sales biliares y no ser sensible a enzimas proteolíticas; tener capacidad de adherirse a superficies epiteliales y un mecanismo específico de adhesión, crecimiento rápido en colon; producir componentes antimicrobianos; tener efecto inmunomodulador sin efecto proinflamatorio y conservarse vivo y estable durante su procesamiento y conservación. Las cepas más utilizadas son Lactobacillus y Bifidobacterium (3).

Los lácteos fermentados son productos lácteos preparados con leche en polvo o concentrada que ha sufrido pasteurización, esterilización o ebullición a la que se inocula Lactobacillus. Son utilizadas en la elaboración de yogures y determinados productos de fermentación de la leche. Presentan un efecto modulador de mucosa intestinal, ejercen un mecanismo preventivo contra infecciones y mantenimiento de la homeostasis del sistema inmune, sin la inducción de efectos perjudiciales, como alergias o reacciones autoinmunes (4). Los estudios llevados a cabo en animales y en humanos se han centrado en los efectos de las leches fermentadas sobre tres funciones del sistema inmune: reconocimiento del antígeno, destrucción del mismo y regulación del material destruido. Así, se ha podido observar que los macrófagos, inmunoglobulinas específicas y algunas citoquinas se modifican tras la ingesta de leche fermentada (5).

El objetivo de nuestro trabajo fue realizar un estudio descriptivo nutricional de una población alérgica y tras una encuesta nutricional, analizar la influencia del consumo de lácteos fermentados en determinados marcadores alérgicos.

\section{PACIENTES Y MÉTODOS}

\section{PACIENTES}

Seleccionamos un total de 44 pacientes alérgicos de una muestra de 15.822 pacientes atendidos en la consulta de alergia del Hospital Río Hortega de Valladolid, y diagnosticados con mismas técnicas. Estos pacientes fueron remitidos a la consulta de Nutrición, realizándose una valoración nutricional y encuesta nutricional, observando si toman productos lácteos y la cantidad (número y ml-mg) a la semana. Además se analizaron diferentes variables relacionadas con su proceso alérgico. A todos los pacientes se les recogió como datos epidemiológicos edad, sexo y lugar de residencia.

\section{VALORACIÓN ALÉRGICA}

Todos los pacientes fueron diagnosticados mediante pruebas cutáneas de hipersensibilidad, utilizando una batería de alérgenos estándar (pólenes, antígenos de polvo, epitelios, hongos, alimentos, controles (suero salino, histamina) y pruebas fraccionadas cuando existe alergia a un alérgeno determinado (UNICAP pharmacia CAP System). Se consideran positivas cuando el diámetro mayor de la pápula es igual o mayor $1 / 100$ de la de histamina control (> $5 \mathrm{~mm}$ ). La segunda prueba utilizada en el diagnóstico es la determinación sérica de los niveles de Ig E específica, se considera elevada por encima de $0,35 \mathrm{Ul} / \mathrm{ml}$ o KU/L. En todos los pacientes se recogieron las siguientes variables; número de brotes alérgicos al año, diámetro del rash cutáneo (prick) y niveles séricos de IgE.

\section{VALORACIÓN ANTROPOMÉTRICA}

A todos los pacientes se les determinó el peso, en una báscula calibrada (100 g), la talla con un tallímetro estándar calibrado $(1 \mathrm{~mm})$ e índice de masa corporal $(\mathrm{IMC})=$ peso $(\mathrm{g}) /$ talla $^{2}(\mathrm{~m})$. Además se les midió el pliegue tricipital (PT), también se determinó la circunferencia del brazo (CB) y circunferencia muscular del brazo (CMB). Para determinar estos parámetros se midió la $\mathrm{CB}$ con cinta métrica calibrada en $\mathrm{mm}$ en el mismo lugar del brazo donde se realizó la medición de PT, la medición se realizó 3 veces y se tomó la media. Para el cálculo final de $\mathrm{CMB}$ se utilizó la fórmula $(\mathrm{CMB}=\mathrm{CB}$ ( $\mathrm{PT} * 0,314)$, expresado en $\mathrm{cm}$. Todos estos parámetros antropométricos fueron medidos siempre por la misma persona, para evitar la variabilidad interindividual en las medidas antropométricas. Se utilizó como tabla de referencia la de Frisancho (6).

\section{ENCUESTA NUTRICIONAL}

Se realizó una encuesta nutricional de 7 días. Este método consiste en recoger la ingesta de nutrientes durante 7 días, todas las encuestas fueron realizadas por la misma dietista. Para realizar esta encuesta se preguntó sobre consumo de alimentos y bebidas, modo de preparación, nombre comercial, ingredientes de la receta y cantidad. Estas encuestas fueron calibradas posteriormente con un programa informático propio de la unidad utilizando las tablas de composición de alimentos nacionales (7).

\section{ANÁLISIS ESTADÍSTICO}

Con los datos obtenidos se generó una base de datos con el paquete estadístico SPSS, para Windows v. 10.0.6. (SSPS Inc., EE.UU.). Se realizó un análisis descriptivo de las variables cualitativas, expresadas en frecuencias (porcentajes) y de las cuantitativas expresadas en media (desviación estándar). Estos parámetros se compararon mediante el test de la t de Student para variables independientes en las paramétricas y el test de Friedman para no paramétricas. El análisis de la distribución normal de las variables continuas se realizó mediante el test de Kolmogorov-Smirnov. Para comparar variables en varios grupos se utilizó el test de Análisis de la Varianza 
(ANOVA), con el test post-hoc de Bonferroni. Las diferencias entre las variables cualitativas se analizaron mediante el test de Chi cuadrado, con correcciones de Fisher y de Yate cuando las condiciones lo requirieron. Se consideraron diferencias significativas $\mathrm{p}<0,05$.

\section{RESULTADOS}

Las características epidemiológicas de los pacientes fueron: $18(40,9 \%)$ eran mujeres y $26(59,1 \%)$ varones con una media de edad de 24,5 (10,3 años). Un total de $14(31,8 \%)$ residían en el medio rural y $30(68,2 \%)$ en la ciudad. Los datos relativos al resto de variables nutricionales antropométricas (peso, talla, IMC, PT, CB y CMB.) se exponen en la tabla I. Se realizó un análisis de Percentiles de PT; CMB y CB utilizando la tabla de Frisancho et al (6), se muestra en la tabla II. La mayoría de los pacientes $(20,5 \%)$ se encuentran en el P25-50 tanto para el pliegue tricipital, como circunferencia del brazo y circunferencia muscular del brazo lo que indica que mantienen un peso e IMC dentro de la normalidad, así como los compaltimentos graso y proteico muscular.

\section{TABLA I}

\section{VARIABLES ANTROPOMÉTRICAS NUTRICIONALES}

\begin{tabular}{lc}
\hline Variable & Media (desviación estándar) \\
\hline Peso $(\mathrm{kg})$ & $68,02(16,03)$ \\
Talla $(\mathrm{cm})$ & $169,58(10,40)$ \\
IMC $\left(\mathrm{kg} / \mathrm{m}^{2}\right)$ & $23,63(3,67)$ \\
PT $(\mathrm{mm})$ & $13,81(5,43)$ \\
CB $(\mathrm{cm})$ & $27,81(4,50)$ \\
CBM $(\mathrm{cm})$ & $23,89(4,03)$ \\
\hline
\end{tabular}

IMC: índice de masa corporal; PT: pliegue tricipital; $C B$ : circunferencia del brazo; CBM: circunferencia muscular del brazo.

\section{TABLA II}

FRECUENCIAS EN CADA GRUPO DE PERCENTILES

\begin{tabular}{lccc}
\hline Percentiles & $T P$ & $C M B$ & $C B$ \\
\hline$<5$ & $4,5 \%$ & $11,4 \%$ & $13,6 \%$ \\
$5-10$ & $0 \%$ & $2,3 \%$ & $2,3 \%$ \\
$10-25$ & $9,1 \%$ & $11,4 \%$ & $13,6 \%$ \\
$25-50$ & $20,5 \%$ & $25,0 \%$ & $27,3 \%$ \\
$50-75$ & $18,2 \%$ & $15,9 \%$ & $13,6 \%$ \\
$75-90$ & $13,6 \%$ & $9,1 \%$ & $9,1 \%$ \\
$90-95$ & $2,3 \%$ & $9,1 \%$ & $6,8 \%$ \\
$>95$ & $0 \%$ & $4,5 \%$ & $2,3 \%$ \\
\hline Total & $100,0 \%$ & $100,0 \%$ & $100,0 \%$ \\
\hline
\end{tabular}

IMC: índice de masa corporal; PT: pliegue tricipital; CB: circunferencia del brazo; CBM: circunferencia muscular del brazo.

La encuesta nutricional mostró como 43 pacientes $(97,7 \%)$ tomaban lácteos no fermentados y sólo un paciente no consumían lácteos; la media de número de lácteos no fermentados a la semana fue de $11,8(6,31)$ y la media de ml-mg de lácteos no fermentados a la semana fue de $22.339,3 \pm 1.199,3$. Con respecto al consumo de lácteos fermentados, 16 pacientes consumían estos productos habitualmente $(27,1 \%)$, con una media de tomas a la semana de $1,79(3,16)$ y una cantidad total a la semana de mg-ml $(593,4(461,9)$. Con respecto a las variables alérgicas, el número de crisis/año fue de $1,44(1,8)$, los valores medios de Ig E séricos fueron de 35,6 $(31,93) \mathrm{Ul} / \mathrm{L}$ y con un valor medio del rash cutáneo de $10,09(2,8) \mathrm{mm}$.

Al realizar el análisis específicamente para los lácteos fermentados, no existieron diferencias significativas entre los pacientes que no consumían lácteos fermentados y los que sí consumían, en el rash cutáneo $[10,4(3,4) \mathrm{mm}$ vs $9,5(1,12)$ mm;ns], en los niveles de $\operatorname{IgE}[32,2(29,2) \mathrm{UI} / \mathrm{L}$ vs 4,24 $(36,7)$ UI/L;ns], ni en el número de crisis $[1,1(1,3)$ crisis/año vs 2,1 $(2,4)$ crisis/año; ns]. Tampoco existió correlación entre los niveles de ingesta de lácteos fermentados y las diferentes variables alérgicas. No obstante, al dividir a la población que consumía lácteos fermentados en dos grupos en función de si estaban o no por encima de la media de consumo del grupo, obtuvimos diferencias significativas en el rash cutáneo, siendo superior en los que consumían menos $(10,12(0,8) \mathrm{mm} v \mathrm{~s}$ $8,85(1,1) \mathrm{mm} ; \mathrm{p}<0,05)$, sin encontrar diferencias en el resto de variables alérgicas. El lugar de residencia no se relacionó con ninguno de los parámetros alérgicos o de consumo de lácteos fermentados.

\section{DISCUSIÓN}

En las dos últimas décadas pasadas, la incidencia de enfermedades alérgicas ha aumentado en países industrializados, sobre todo en la infancia; los factores causales están relacionados con el estilo de vida como la mejora de condiciones de higiene, disminución del tamaño familiar y de muchas infecciones (vacunaciones, tratamientos precoces e intensos, alimentos y ambientes estériles) disminuyendo la exposición a microbios en etapas tempranas de la vida, lo que evita que los niños desarrollen sus defensas. Así se ha observado menor número de alergias en familias numerosas, en poblaciones con pruebas tuberculínicas positivas, vacunadas con BCG o que sufrieron epidemias de sarampión.

El papel beneficioso de las leches fermentadas para la salud se conocía desde hace varios siglos, pero no fue hasta 1908 cuando el científico ruso Ilya Metchnukoff enfatizó los beneficios que proporcionaba el consumo de yogur a los pobladores de los Balcanes, en los que asoció su gran longevidad y buena salud física al elevado consumo de yogur, por sus investigaciones recibió el premio Nóbel de Medicina en ese año. Ha sido probada in vitro o in vivo el efecto de probióticos en estados patológicos como diarrea, vaginitis, infecciones del tracto urinario, desórdenes inmunológicos, intolerancia a la lactosa, hipercolesterolemia y alergia alimentaria. Sobre sistema inmune, ciertas cepas de LAB, actúan sobre reacciones de hipersensibilidad retardada, producción de anticuerpos, activación funcional de macrófagos, algunas son capaces de prevenir infecciones y ejercer acción antitumoral al inhibir agentes químicos carcinogénicos.

En las alergias se empieza a estudiar los efectos de las LAB, sin embargo hasta el momento los resultados son controvertidos y no se conocen los mecanismos de acción. Se ha observado remisión de sintomatología de alergias alimentarias tanto síntomas gastrointestinales como otros sistémicos como eczema o dermatitis atópica. También se ha visto una disminución significativa de marcadores intestinales y sistémicos de origen alér- 
gico. En nuestro trabajo, el grupo de pacientes con un consumo de más de $593 \mathrm{mg} / \mathrm{semana}$ de lácteos fermentados mostró una disminución en el diámetro del rash cutáneo, sin mostrar repercusión en otras variables alérgicas. En otros estudios de intervención, se ha demostrado la remisión de síntomas alérgicos de tipo nasal en una población de 42 jóvenes y 56 adultos, tras la ingestión de 200 gramos al día de yogur durante un año (8), en comparación con un grupo control. Sin embargo, no se sabe cuáles podrían ser los mecanismos implicados, puesto que los autores no han encontrado diferencias significativas en los parámetros inmunológicos estudiados. En otro trabajo, con diseño de tipo cross-over, en el que individuos asmáticos sometidos a una ingesta de $225 \mathrm{~g}$ de yogur 2 veces al día con o $\sin L$. acidophilus durante un mes, se ha encontrado en el grupo que consume el yogur con L. acidophilus que la eosinofilia es menor, pero no aparecen diferencias significativas en la clínica de los pacientes, ni en su calidad de vida (9). Del mismo modo, cuando se ha estudiado en pacientes atópicos los efectos del yogur, conteniendo L. bulgaricus y Streptococcus thermophi- lus, no se ha observado ninguna mejoría significativa en los parámetros inmunológicos estudiados (función fagocítica y respuestas inmunes humoral y celular) (10). Isolali y cols. estudiaron infantes que presentaron eczema ligado, exclusivamente a lácteos. Ellos aleatoriamente recibieron una fórmula de proteína hidrolizada con y sin probióticos. Se observó una resolución completa del eczema en pacientes que recibieron la suplementación, por un aumento de la citoquina IL10 (11). En otro reciente estudio en jóvenes con alergia al polen y a la manzana, con síntomas intermitentes atópicos y/o asma ligero, no se observa mejona tras 5,5 meses de suplementación con Lactobacillus Rhamnosus. Sin embargo, los autores concluyen que podría ser debido a una muestra pequeña (12).

En conclusión, el consumo de lácteos fermentados en pacientes alérgicos puede ser beneficioso, disminuyendo determinados marcadores alérgicos. No obstante, son necesarios estudios con mayor número de pacientes y de intervención para evaluar esta influencia sobre variables clínicas de mayor relevancia.

\section{Bibliografía}

1. Sociedad Española de Alergia e Inmunologia Clínica. Las enfermedades alérgicas respiratorias en Atención Primaria: Diagnóstico y Tratamiento etiológico. Barcelona: Astra S.A. 1988; 31 (45); 59-81.

2. Fuller R. Probiotics in man and animal. J Applied Bacteriol 1989; 66: 365-378.

3. Fuller, R. Probiotics. The Scientific Basis, Champan \& Hall, 1992.

4. Kurmann JA, Rasic JL. In: Therapeutic Properties of Felmented Milks (Robinson RK, ed.) 1991. p. 117-57.

5. Solis-Pereyra et al. Interferon induction by Lactobacillus bulgaicus and Streptococcus themphilus in mice. Eur Cytoquine Net 1991; 2: 299-303.

6. Tablas de percentiles de Frisancho. Medida de Pliegue tricipital.

7. Mataix J, Manas M: Tablas de composición de alimentos españoles. 33. Ed: University of Granada, 1998.

8. Trapp CL, Chang CC, Halpern GM, Kent CL, Gershwin ME. The

influence of chronic yogurt consumption in population of young and elderly adults. Int J Immunother 1993; IX: 53-64.

9. Wheeler LG, Shema SJ, Bogle MI, et al. Immune and clinical impact of Lactobacillus acidophilus on asthma. Ann Allergy Asthma Immunol 1997a; 79: 229-33.

10. Wheeler JG, Bogle ML, Shema SJ, Shirrell MA,Stine AK, Helm RM. Impact of dietary yogurt on immune function. AM J Med Sci 1997b; 313: 20-123.

11. Isolauri E, Arvola T, Sutas Y, Moilanen E, Salminen S. Probiotics in the management of atopic eczema. Clin Exp Allergy 2000; 30: 160410 .

12. Helin T, Haahtela S, Haatela T. No effect of oral treatment with an intestinal bacterial strain Lactobacillus rharnnosus (ATCC 53103), on birchpollen allergy: a placebo-controlled double-blind study. Allergy 2002; 57: 243-246. 